\title{
Computational Intelligence Applied to Monitor Bird Behaviour
}

\author{
D. F. Larios ${ }^{1}$, C. Rodríguez ${ }^{2}$, J. Barbancho ${ }^{1}$, M. Baena ${ }^{3}$, F. Simón ${ }^{1}$, \\ J. Marín ${ }^{2}$, C. León ${ }^{1}$ and J. Bustamante ${ }^{2}$ \\ ${ }^{I}$ Department of Electronic Technology, University of Seville, Seville, Spain \\ ${ }^{2}$ Department of Wetland Ecology, Doñana Biological Station (EDB-CSIC), Seville, Spain \\ ${ }^{3}$ ICTS, Singular Scientific and Technological Infrastructure, Doñana Biological Station, Seville, Spain
}

\begin{abstract}
Keywords: Neuronal Network, Computational Intelligence, Data Fusion, Environmental Monitoring, Sensor Networks.
Abstract: The best way to obtain relevant information about the behaviour of animals is direct observation (of individuals). However, traditional close-up observations can interfere on the behaviour, and taking biometric measurements requires the capture of individuals, which also causes stress. This paper describes an automatic motoring system for birds breeding in nest boxes. The main goal is to significantly increase the amount and quality of data acquired on bird behaviour without stressing the individuals or interfering. This system is based in an interconnected embedded sensor network, which permits sharing this valuable information with researchers all over the world through the internet. Each device of the network is a smart nest-box that allows a cross-validation of sensor information and data quality. This system has been evaluated for the specific case of a lesser kestrel breeding colony in Southern Spain. The lesser kestrel is an insectivorous migratory falcon that readily accepts nest-boxes. The system has been named HORUS and the results obtained from a year experiment demonstrate the efficiency of this approach.
\end{abstract}

\section{INTRODUCTION}

For zoologists, one of the most important periods for a bird is the breeding period, being this period one of the most frequently studied. Mate acquisition, nest defence, mate feeding, incubation, and chick rearing (including provisioning flights) are studied in the surroundings of the nest. Many of these aspects help us understand key topics in ecology, such as what factors influencing lifetime reproductive success (LRS: Newton, 1992), the parent-offspring conflict (Trivers, 1974; Schlomer et al., 2010), or evolutive stable strategies (ESS: Maynard-Smith and Price, 1973) regarding sex roles in reproduction (Kokko and Wong, 2007).

Classic monitoring methods that require the capturing of individuals or close-up observations limit the amount and quality of data that can be obtained. Therefore, in this paper a remote monitoring system based on smart nest-boxes is proposed. These smart nest-boxes allow acquiring high amount of data without stressing the animals, gathering long-term and highly reliable information on the species.

The proposed system, called HORUS, permits gathering basic information on the identity of individuals, studying its behaviour and the temporal changes in individual body mass. All this information can be made accessible through the internet to scientist all over the world.

Some of the information recorded by the system can be used to study, without interference, the behaviour of species during the breeding period. One of the most important biometric parameters in birds is body mass. It allows us to measure the impact of parental care on breeding individuals. Manually, it is impossible to develop a continuous monitoring of this parameter. Capturing causes too much stress on the individual in its most sensitive period. Another important parameter is the measurement of the amount of food brought to the nest by individuals to feed their offspring.

The proposed system allows us to perform a continuous monitoring of the reproduction without stressing the individuals, e.g. obtaining reliable body mass measurements every time a bird enters or leaves the nest. The main problem obtaining the measurements is the movement of the animal, which produces unstable values. These values have been calibrated using a neuronal network processing, 


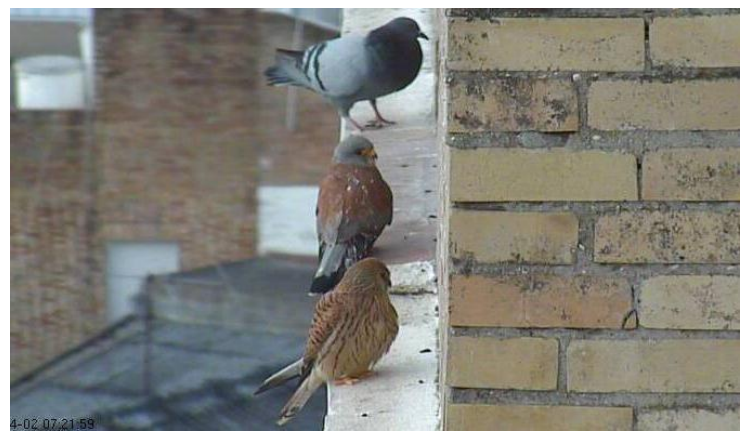

Figure 1: Picture automatically taken by the outside camera of the monitored colony with the HORUS external sensors. From top to down it shows a pigeon, a lesser kestrel male and a female.

obtaining high accurate measurements.

The rest of the paper is organized as follows: Section 2 focuses on the lesser kestrel behaviour study, especially comparing a traditional approach versus automated data reading. Section 3 briefly describes the HORUS system infrastructure. A detailed description of the information treatment developed in this project can be found in section 4 . The results obtained with our system are shown in section 5. Finally, section 6 sum-up conclusions and provides remarks.

\section{LESSER KESTREL BREEDING BEHAVIOR STUDY}

The lesser kestrel (Falco naumanni, figure 1) is a small (body mass around 150 grams) migratory falcon inhabiting open landscapes (Cramp and Simmons, 1980). It is a colonial species that breeds in old buildings, such as churches or castles within urban areas in Western Europe. The species experienced a marked decline in its Western Palearctic breeding range in the middle of the 20th century (Cramp and Simmons, 1980; Biber, 1990). Considered previously one of the most abundant raptors in Europe (Bijleveld, 1974) the lesser kestrel became extinct in several countries (e.g. Austria, Hungary, Poland) and practically disappeared in others (e.g. France, Portugal, Bulgaria).

Mediterranean Spain constitutes its stronghold in the Western Palearctic (Biber, 1990). However, the Spanish population also suffered a precipitous decline, as it dropped from an estimated 20,00050,000 pairs in the 1970 s (Garzón, 1977) to 4,0005,000 breeding pairs in 1988 (González and Merino, 1990). This decline has been attributed to the reduction in both the extent and quality of foraging habitats (Peet and Gallo-Orsi, 2000). The species is also sensitive to climate warming (Rodriguez and Bustamante, 2003). So it makes for a good model species to study the impact of global change on an endangered species.

\subsection{Traditional Monitoring}

The "Estación Biológica de Doñana" (EDB-CSIC) has been monitoring lesser kestrel colonies since 1988. It has been recording colony occupancy and breeding success in terms of number of fledglings and proportion of successful nests. Regarding individual monitoring, birds have been marked with metal and PVC rings with a unique alphanumeric code that allows identifying individuals by using telescopes. Biometric measures were taken sporadically for all marked individuals when captured. Due to ethical reasons, however, the number of captures in the nest is limited (the capture alters breeding behaviour and may jeopardize the survival of the offspring) and the majority of resightings were made with telescopes. This causes high differences in the frequency of recaptures among individuals mainly due to differences in detectability. In a classic data base monitoring, 2,135 birds figured as recaptured (including resightings with telescope). On average they were captured 3 times on the same breeding season (range: 1-70). In approximately $45 \%$ of cases, body mass was measured and maximum number of measurements per bird and year was 4 .

Because of that, the pattern of body mass variation of breeding adults from arrival to the colony in mid-February to the end of the nestling period in mid-July is not well known. Accordingly, we have no information on the pattern of intraday variation in body mass.

The proposed remote monitoring system aims to bridge the above detailed logistic and ethic gaps, thus allowing us to get enough information to document both patterns.

\subsection{Automated Data Reading}

Habitat monitoring has evolved greatly evolution due to the boom of sensor networks technology.

Several consequences have been caused due to the increase of sensors: Firstly the quality of information grows in time and on the spatial domain; secondly the possibility of transmitting the measured data through the network increases the need of having high bandwidth communications; and thirdly, it to the reduction of the cost of the data 


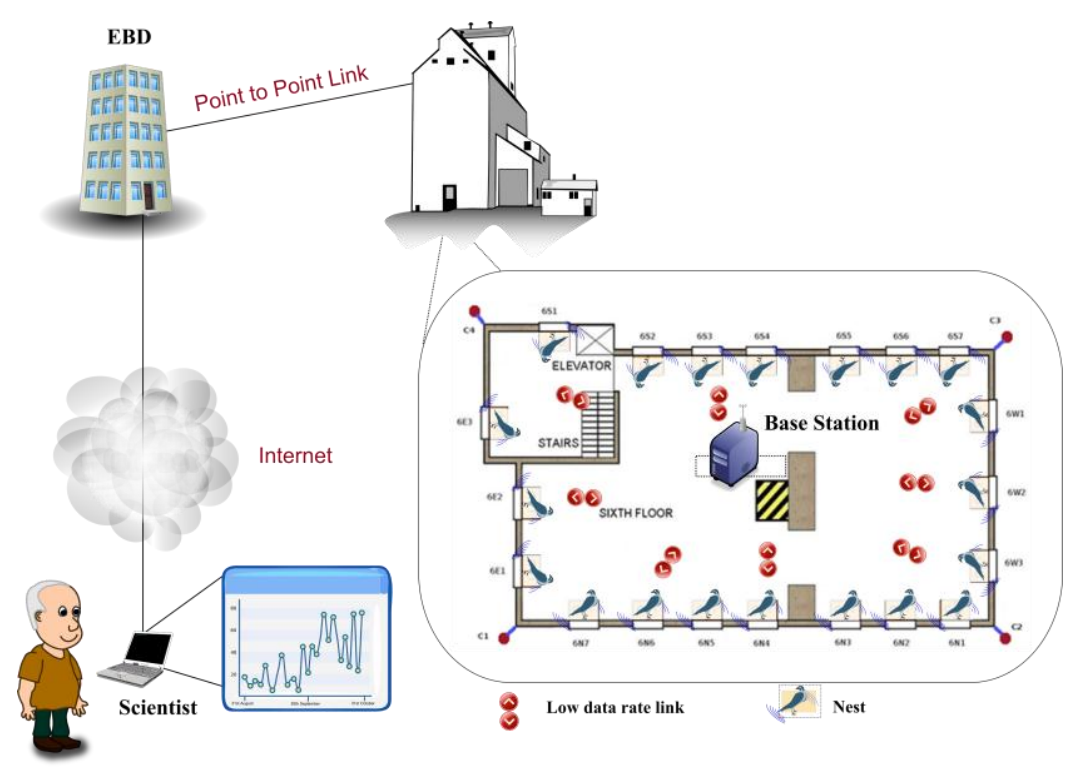

Figure 2: HORUS Network scheme.

storage makes possible to save huge amounts of data.

All these consequences imply some negative effects: an increase of data traffic and increase of power consumption.

Some authors have taken these effects into account (Cook, 2007; Sridhar, 2007) and have expressed the need to employ processing techniques in order to reduce these handicaps.

There are different approaches to habitat monitoring. Some of them use wireless sensor network technology in order to acquire and process the physical information (García-Sánchez et al, 2010; Handcock et al, 2010; Valente et al, 2011; Carullo et al, 2009). Others focus on the needed middleware that allows access to the physical information (Hwang et al., 2010; Farshchi et al., 2007).

In our approach, both aspects are considered.

\section{HORUS INFRAESTRUCTURE}

The proposed infrastructure is a distributed system as figure 2 depicts. This figure shows the most important devices of the proposed architecture.

These devices will now bebriefly described.

\subsection{Network Infrastructure}

The HORUS infrastructure is made up of different subsystems interconnected through a low data rate communication network.
This network has been designed considering the following restrictions:

- The devices that provided information to the network are deployed in a spread way without any previous planning.

- The data rate associated with the data sources is low $(<250 \mathrm{kbps})$

- The system could be easily scalable.

The network used in HORUS can be accessible through different physical media (wireless or wired based).

Robustness of the network is very important, for the proposed application: during the breeding period, it is not possible to realize maintenance tasks, because it can disturb and stress the colony. All detected failures would be repaired in winter, after the birds have left the colony.

\subsection{Base Station}

The process server is a system that offers the following services:

- Database server.

- Monitoring and control system.

- Remote control access.

The database stores all the historic sensors information gathered from the system.

The monitoring control system is a program responsible for adding additional valuable information to the sensor measurements, such as information about the nest sender, a time stamp register or a control sequence, that permits 


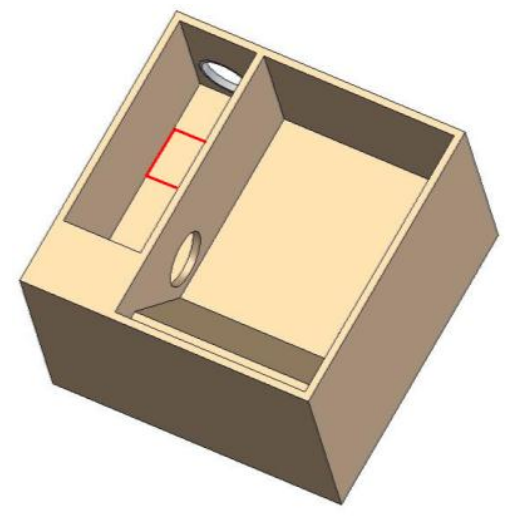

Figure 3: Nest cabinet.

determining the number of loss packets. This system stores the information in the database.

Remote access control offers the cloud services for remote users, such as biologists. These services permit remote access to the sensors database.

\subsection{Smart Nest-Boxes}

The smart nest-boxes are the main components of the monitoring systems. It consists of the next two blocks:

- The nest cabinet.

- The electronic system

The nest cabinet (Figure 3) is divided into two parts: a corridor and the incubation chamber. This nest cabinet has a smart design to ensure that the birds pass the corridor each time they enter or leave the nest. The advantage of this is to allow the distribution the sensors in a small area (the corridor) where the animal is forced to pass and, therefore, it ensures obtaining the sensor information.

The electronic system (Figure 4) of each smart nest-box is accomplished with the next subsystems:

\subsubsection{Microcontroller Board}

This board is based on the ATmega2560, an economic, low power and robust microcontroller. It controls and processes the nest's sensor information. This board communicates with sensors and other components, and processes the collected information that is sent to the process server over the communication interface.

The program implemented in the microcontroller performs the following tasks:

- Communicates with the process server over a communication interface, and synchronize clock time with this.

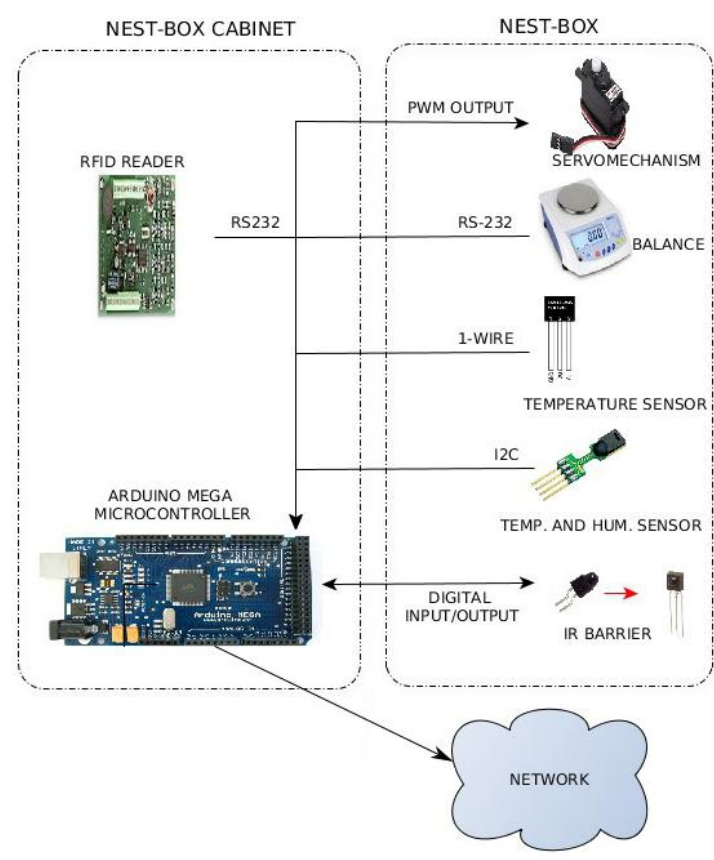

Figure 4: Architecture of the electronic system.

- Checks infra-red barriers. Each nest-box has two infra-red barriers at both extremes of the corridor. The sequence in which they are activated indicates whether birds enter or leave the nest-box.

- Checks if the RFID reader has read a code from ringed kestrels.

- Obtains the body mass measurement from a digital balance.

- Reads the temperature and humidity of the nest.

- Controls the RFID reader to identify individuals.

\subsubsection{Sensors Board}

A sensor board adapts the logic levels from the nest sensors to the microcontroller board's requirements.

All the nest's sensors are spread onto the corridor of the nest. Positions of sensors are designed to ensure that every time the birds pass the corridor the system registers at least one record per sensor.

The deployed sensors are:

- A digital balance. It allows a maximum weight of $600 \mathrm{gr}$. and an accuracy of $0.01 \mathrm{gr}$, offering 16 measures per second. It permits getting an estimate of the body mass of the individuals in movement. Although the pan is round, it has been modified to be rectangular in order to fit the shape of the corridor. 


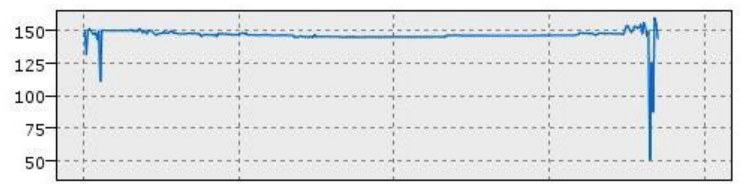

(a)

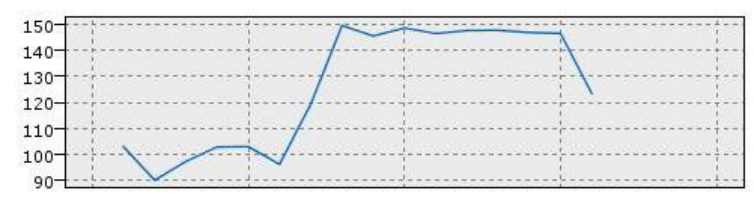

(c)

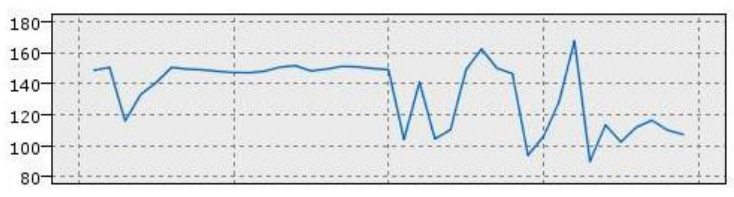

(b)

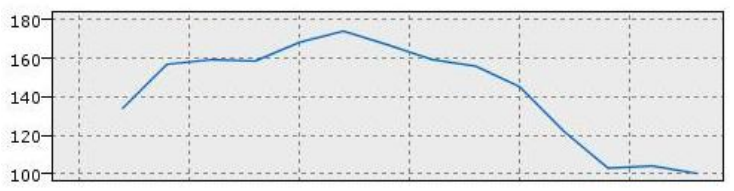

(d)

Figure 5: Different weight pattern Y-axis, weight in grams. X-axis, samples.

- An integrated temperature sensor located in the window. It is calibrated to operate in environmental temperature range. It is used to measure the nest temperature.

- An integrated humidity sensor. It is used to measure the nest humidity.

- Two infra-red barriers, used to trace the direction of birds' movements.

- A RFID reader. It communicates via RS-232C and offers a reading on the unique ID of a tagged bird, when it is passing through the nest entrance. This system has mechanisms to avoid collisions, permitting operation even when there are several birds around the entrance.

- A Servomechanism. It is used to remotely capture birds when they enter in the nest-box.

\section{TREATMENT OF THE INFORMATION}

As described before, every nest-box provides the following information:

- Measurements of body mass: The digital balance used offers 16 measurements per seconds without calibration and classifies measurements as stable or unstable.

- IR information: These sensors permit determining if the birds go into or go out of the nest.

- RFID information: It permits attributing the information of other sensors to an individual bird.

All this information is obtained from the sensors deployed in every nest-box. The sensors offer relevant information on the individual breeding at the colony to the biologists that study them. This information, except the body mass, cannot be added, as they inform about discrete events. Therefore, this information is sent directly to the database without any local processing or treatment.

On the other hand, the digital balance offers a high amount of information. Its frequency of measurement is much higher than the body mass evolution of the animal. i.e., the animal body mass evolution has more inertia than the weight provided by the balance. Due to this, it is possible to perform a data pre-processing about weight information, reducing with that the amount of information send to the central processing.

\subsection{Weight Pre-processing}

The algorithm described in this paper, is focused on locally pre-processing the weight information, to reduce the amount of unnecessary information and increase its accuracy. It is designed to be executed in each nest-box, in the microcontroller board. It has been designed to fulfil the next goals:

- To reduce the amount of useless information in the database using local pre-processing.

- To increase the accuracy of the measurements, calibrating the results obtained.

- To increase accuracy of the communication network, reducing the amount of packet loss, the delays and the collisions.

- To increase the amount of useful information in the database, estimating a body mass from each pattern with non-stable measurements.

- To permit its execution on devices with low resources.

To increase the accuracy a tare calibration is necessary. The balance used offers measurements without a tare calibration. This calibration would be obtained consulting the body mass measured by the balance, when there is no animal on the pan i.e., when the measured weight is below a certain 
threshold. This threshold can be obtained as a function of the body mass of the animals to monitor. In our deployment for the Lesser Kestrel (with a body mass range of 100-190 g) a threshold of 100 grams of has been used.

In the real deployment we have proven that the tare does not change significantly during a year. Therefore, measuring the tare only once per day offers enough accuracy for the proposed system.

On the other hand, as described before, the balance offers 16 weight measurements tagging them every second by itself as stable (i.e., measurements that remain a same value during a long period of time) or unstable. But birds usually do not pass over the balance slow enough to obtain stable measurements. This causes the database to have a high amount of the information as unstable measures. In the real prototype only about $15.25 \%$ of the measured patterns had a stable measurement, considering a pattern as the collection of measurements obtained from the time the bird gets on the balance (i.e., when the balance acquires a weight over the threshold) until the animal gets out of balance (i.e., when the balance acquires during 5 seconds weights below the threshold). Fig. 5 shows different examples of weight patterns obtained in the real deployment with these conditions.

This figure shows different real weight patterns obtained from the same animal in different days. Only pattern (a) has some stable measurement. These stable measurements have been compared with measurements of the animal done manually capturing the bird. The stable tare measurements are correct, but not frequent enough to obtain a long term sequence of body mass temporal change of the birds at the colony.

To solve this, a computational intelligence algorithm to estimate the body mass of animals from the patterns with non-stable weights has been developed, increasing the amount of useful information. This neuronal network algorithm is described below.

Initially, the system has been designed to store, in the central server, all weight measurements of the pattern acquired by the balance, stable or unstable, but it causes high bandwidth consumption in the communications interface.

To reduce the amount of useless information, the proposed algorithm only sends one estimated weight to the database for each measured patterns. If the pattern has some stable measurements, the estimated weight sent to the central server will be the average of the obtained stable measurements. If no measurements of the pattern are stable, the weight is estimated through a computational intelligence algorithm. In both cases, only one selected weight per pattern is sent to the database. These selected weights are calibrated with the tare, before sending them.

The proposed algorithm is summed-up in the next pseudo-code:

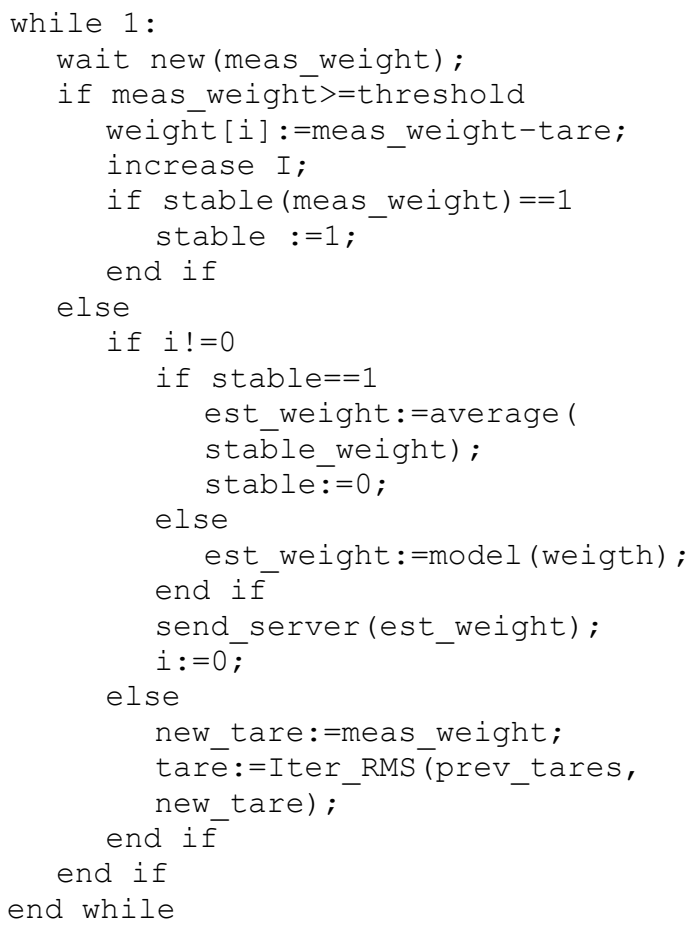

\subsubsection{Applying Machine Learning for Weight Recognition}

For this application, an algorithm has been evaluated. Initially, an algorithm without machine learning based on the differences between consecutives measurements has been considered. This algorithm considers a weight stable if there are more than a certain number of measurements of the same weight. This is similar to the internal algorithm of the balance for tagging measurements as stable or unstable, but it is less restrictive: the balance requires a high number of measurements with the same value to consider a measurement stable. It permits the retrieval of some weights from the unstable patterns, but it fails with complex patterns. Our proposal of using computational intelligence (machine learning) increases the percentage of success.

Machine learning is widely used in pattern recognition, but its use in animal monitoring is less widespread. Other supervised learning techniques apart of the neuronal network have been considered. 
Non supervised techniques, such as Self-Organized maps (SOM, Kohonen, 1990) or Support Vector Machine (SVM, Cortes 1995) were discarded, because we have some stable measurements that permit performing training.

One example of the considered supervised machine learning techniques is the use of Artificial Neuro-Fuzzy Inference Systems (ANFIS; Jang, 1993). ANFIS has many applications in the evaluation of complex systems, but it requires a previous knowledge of the system to design the rules and the initial system. This system was discarded; due to the complex forms of the patterns that do not easily permit acquire this initial system.

Expert systems or case based experts system were not considered, due to the amount of previous information gathered from the smart nest-box was not sufficient for these kinds of systems.

For these reasons, a neuronal network model was finally chosen. The variables used as inputs of the model are as follows:

- Max 1: The most repeated weight in a pattern (the largest if multiple).

- N_1: Number of repetitions of the previous variable in a pattern.

- Max_2: The second most repeated weight in a pattern (the largest if multiple).

- N_2: Number of repetitions, in a pattern, of the previous variable.

- Max_C1: The most consecutively repeated weight in a pattern.

- NC_1: Number of repetitions of the previous variable in a pattern,

- Max_C2: The second most repeated weight, consecutively, in a pattern.

- NC_2: Number of repetitions, in a pattern, of the previous variable.

- N_EL: Total number of weight measures in a pattern.

In order to obtain these parameters, a pattern with at least 5 weight measurements is needed. As

Table 1: Analysis of the database.

\begin{tabular}{|c|c|}
\hline Caption & Value \\
\hline Measurement weight & 2583565 \\
\hline Number of pattern & 51517 \\
\hline Patterns with stable weights & 7856 \\
\hline Average pattern time & 23,18 seconds \\
\hline Days of test & 399 days \\
\hline
\end{tabular}

an value output, the neuronal network model offers a value, called "Output weight". This output reflects the estimated weight of the neuron model and it is the information sent through the network to the server database.

The steps execution of this neuronal network model is summed-up in the next pseudo-code, where the neuronal network is the execution of a three layer network.

Neuronal network needs a set of parameters for its training. These sets have been obtained for each pattern with stable measures, by executing the following steps:

Step 1: A variable name "Target weight" was defined for every pattern. This variable stores the average value of all stable weights. This is the target result of the training of the neuronal network.

Step 2: For every pattern, a new pattern has been created, eliminating all stable measurements.

Step 3: The inputs have been obtained from this new pattern without stable values.

Step 4: The input values for each pattern were stored, together with their respective Target weight into a table, named "Training information"

With these tables two sets of information were obtained, one for training and the other for evaluating the accuracy of the system. In total, the training information table has 1163 sets of values. $50 \%$ of these values (randomly selected) were used for training, and the other $50 \%$ were used for validation.

\section{SIMULATION, TESTS AND RESULTS}

The results obtained with this system can be classified in two types: analysis of the network performance and weight estimation accuracy obtained with the real deployment.

This section summarizes these two types of results.

\subsection{Network Performance}

During the first year of the deployment (2010), the prototype was sending information from all sensors, even the 16 records per second of the balance, to the database of the central server. The main characteristic of the gathered information in the database is summarized in table 1 .

After a year of deployment, the analysis of data allowed us to detect some network conflicts. For example, if different nest-boxes are acquiring weights from individuals at the same time, they are competing for control of the bus, causing data collisions and delays in transmitting information. 
Table 2: Cost per message with CC2420 Radio transceiver.

\begin{tabular}{|c|c|}
\hline Caption & Energy (J) \\
\hline Without data fusion & 255.3 \\
\hline With data fusion & 0.608 \\
\hline
\end{tabular}

The proposed system allows avoiding these conflicts, using the proposed data fusion.

In this section we are going to quantify the advantage of data fusion against the classical centralized systems. Due to that, in this kind of applications it is important to reduce the use of bandwidth as much as possible.

The analysis of database information has been summed-up in the table 1 . It shows that only a $15.25 \%$ of the acquired patterns have any stable measurement.

Knowing that the balance offers 16 Samples Per Second (SPS), the average payload of the application layer per pattern of the system without data fusion can be obtained with the equation 1 .

$$
N_{T, \text { raw }}=16_{S P S} \cdot P_{T} \cdot N_{\text {Bytes }} \cdot N_{\text {msg }}
$$

Where $N_{T \text {,raw }}$ is the number of bytes to send per day at application layer; $P_{T}$ is the length of the pattern in seconds; $N_{\text {Bytes }}$ is the number of bytes to send. 16 bytes in this case and $N_{m s g}$ is the number of messages per day.

On the other hand, with the proposed algorithm, only one message per pattern is sent. In this case, the payload per pattern can be obtained according to equation 2.

$$
N_{T, \text { raw }}=N_{\text {Bytes }} \cdot N_{\text {msg }}
$$

This shows that the amount of information sent to the database varies in function of the number of patterns and the length (in time) of the pattern. Figure 6 depicts these results.

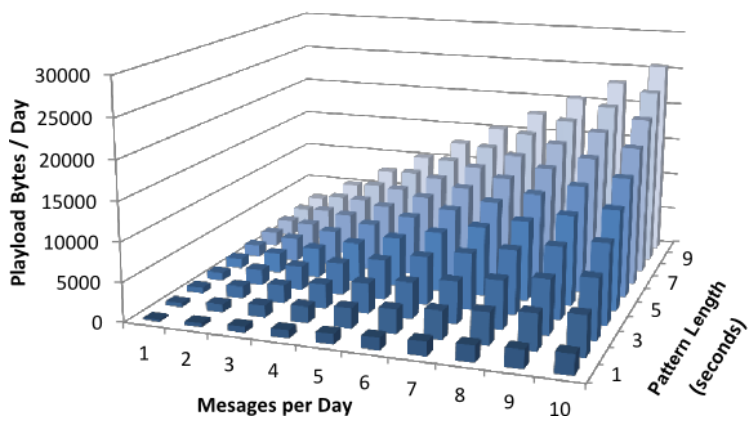

(a)
Concluding, the local processing permits one to drastically reduce the used throughput of the network, especially in days with a high number of patterns.

This data fusion and aggregation scheme is especially important for its use in low bandwidth systems, due to it permitting one to save energy. With the proposed system, only one message per pattern is sent, instead of 16 measurements per second during the capture of the pattern. These results are summed-up in table 2 . They consider the average pattern length of 23.18 seconds, i.e. the average time while the bird is on the balance.

With these conditions and with the CC2420 radio transceiver, widely used in wireless sensor network, permit saving $99.76 \%$ of the energy used in data transmissions, considering a power consumption of $38 \mathrm{~mW}$ in transmission mode (Polastre et al, 2005.

Using all weight patterns obtained in the year 2010 , the proposed body mass estimation algorithm permits the retrieval of around $56.21 \%$ of the patterns without stable measurements.

This is a good result that permits us to obtain an average of 4 body mass estimations per day and nest, which is 4 times higher than using only patterns with stable measurements. It permits to have a continuous tracing of body mass in individuals.

As a conclusion, the local processing permits us to drastically reduce the used throughput of the network, especially in the days with a high number of patterns.

\subsection{Body Mass Estimation Accuracy}

Based on the training and verification set described in section 4.1.1, some analysis has been done to the proposed algorithm for body mass estimation.

With the evaluation set, the system offers an accuracy of $98.7 \%$, i.e., an error in the order of 2

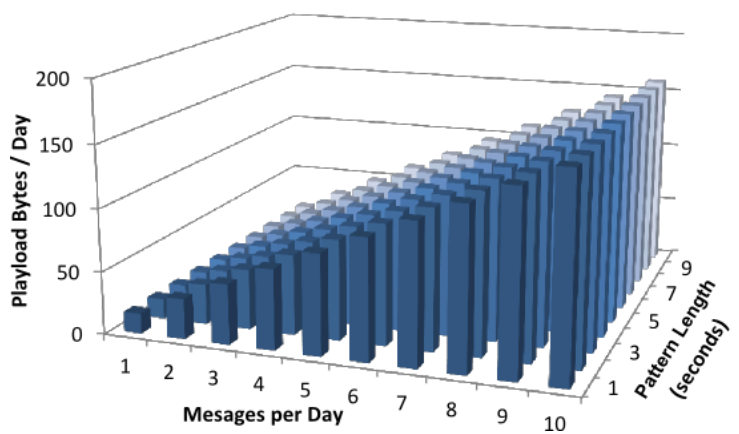

(b)

Figure 6: Bytes per day send, at application layer, to the central server. (a) Without data fusion. B) With data fusion. 


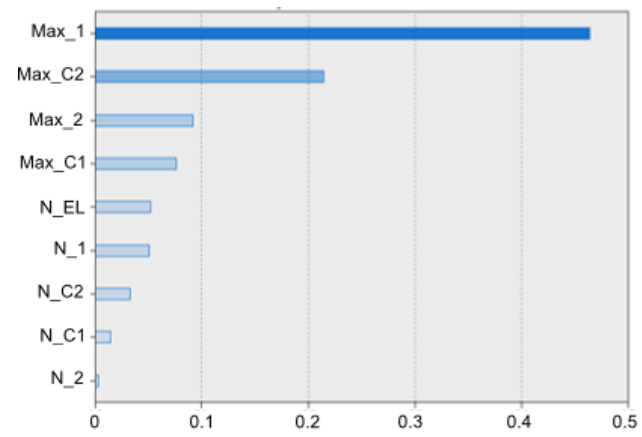

Figure 7: Neural network model: importance of the variables in the calculation of the estimated weights.

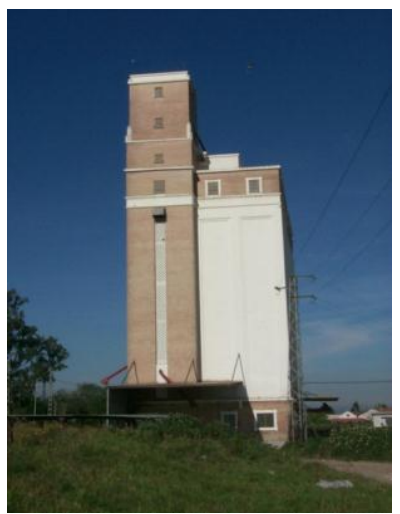

Figure 8: Grain elevator used for the prototype installation.

grams, which is quite small considering the typical body mass of these animals (150 grams).

This accuracy permits analysis of a long series for the evaluation of temporal changes in body mass, and sometimes to determine the body mass of prey, when birds bring medium-sized animals to the nest to feed the nestlings.

From the training procedure, an analysis of the importance of the input parameters in relationship with the target body mass can be obtained. Figure 7 shows these results. This analysis concludes that the selected parameters are valid to effectively estimate the body mass of animals.

\subsection{Real Deployment}

A prototype, for a real validation of the proposed system, has been deployed in the grain elevator of "La Palma del Condado (Huelva Province, SW Spain" (figure 8). At this site, researchers of the Estación Biológica de Doñana have been studying the lesser kestrel colony since 1994. At this colony, kestrels nested on the windowsills of the grain elevator that are sheltered and sufficiently enclosed to make a suitable nesting site.

For the prototype installation we select the windows on the 6th floor of the building where smart nest-boxes were installed, and readily accepted, by kestrels during preliminary checking (3 and 4 nest-boxes during 2008 and 2009, respectively) and also when the definitive prototype installation was made in 2010.

Nest-boxes are placed in all the windows along the sixth floor. They are named " $6 \mathrm{XY}$ ", where $\mathrm{X}$ refers to the cardinal point and $\mathrm{Y}$ is an ordinal number. Each box has two separate entrances and two incubation chambers (I. left and D: right) in a symmetrical distribution. Entrances are placed at the extremes of the box to avoid potential aggressions between neighbours, thus maximizing the number of potential breeding pairs. Nonetheless, the right part has not been opened yet.

The results provided by the system are still being analysed by biologists. However, in its current state, it is possible to obtain some conclusions:

- 18 of the 20 installed nests-boxes were used by breeding kestrels. This leads to the conclusion that the proposed system effectively allows one to gather a high amount of information about the behaviour of breeding individuals without stressing them. If the nest and its sensors were hostile, it would not have been chosen by lesser kestrels breeding pairs.

- A preliminary result of the continuous weighting of individuals will allow the researchers to estimate the cost of breeding in terms of body mass. This cost is directly associated with the foraging trips to feed the nestlings.

- The lesser kestrel mainly feeds on insects, but sometimes can catch slightly bigger prey, such as small rodents, birds or lizards (with around a dozen of grams). The proposed system would permit an analysis of the frequency of big prey captures.

\section{CONCLUSIONS}

The main goal of the proposed system is to use current technological advances in a real-world application in the area of Biodiversity Conservation to study how global climate change could affect a colonial and endangered bird species.

The results obtained conclude that the proposed system would permit its use in a system with low resources and with a low bandwidth usage.

The prototype deployed in Spain for evaluation 
with the lesser Kestrel, has been demonstrated to be a good method for studying these animals. The proposed system permits us to do this evaluation without stressing the animals and without the need of a human observer. As a consequence, this monitoring does not change the animal behaviour, offering reliable information to researchers all over the world that can access the information in real time through the Internet.

The authors are currently working on several improvements of the project: Increasing the number of sensors, such as adding optical barriers over the balance, with the goal of increasing robustness of the body mass measurements; Studying the way to increase the amount of pre-processing, using the rest of the sensors, and looking for a simplification in the use of the system information for the final user.

\section{ACKNOWLEDGEMENTS}

This work has been supported by the Consejería de Innovación, Ciencia y Empresa, Junta de Andalucía, Spain, through the excellence projects HORUS 2006 (reference number P06-RNM-01712), HORUS 2009 (reference number P06-RNM-04588) ARTICA (reference number P07-TIC-02476), and eSAPIENS (reference number TIC-5705). The authors would like to thank the Cámara Agraria de La Palma del Condado, Consejería de Agricultura y Pesca de la Junta de Andalucía, Reserva Biológica de Doñana ICTS y Espacio Natural Doñana (Almonte, Huelva) for their collaboration and support.

\section{REFERENCES}

Biber, J. P., 1990. Action plan for the conservation of westernLlesser Kestrel Falco Naumanni Populations. $I C B P$, Cambridge.

Bijleveld, M., 1974. Birds of prey in Europe. Macmillan Press, London.

Carullo, A., Corbellini, S., Parvis, M., Vallan, A., 2009. A Wireless Sensor Network for Cold-Chain Monitoring. IEEE Trans. on Instrumentation and Measurement, vol.58, no.5, pp.1405-1411, May 2009.

Cook, D. J., 2007. Making Sense of Sensor Data. IEEE Pervasive Computing, pp. 105-108, April-June.

Cortes, C., Vapnik, V., 1995. Support-vector networks. Machine Learning, Springer, vol. 20, pp. 273-297

Cramp, S. \& Simmons, K. E. L. (1980) Handbook of the Birds of Europe, the Middle East and North Africa. Oxford University Press, Oxford

Farshchi, S., Pesterev, A., Nuyujukian, P. H., Mody, I., Judy, J. W., 2007. Bi-Fi: An Embedded Sensor/System Architecture for Remote Biological Monitoring. IEEE Transactions on Information Technology in Biomedicine, vol.11, no.6, pp.611-618,
Nov. 2007.

Garcia-Sanchez, A.-J., Garcia-Sanchez, F., Losilla, F., Kulakowski, P., Garcia-Haro, J., Rodríguez, A., López-Bao, J.-V., Palomares, F., 2010. Wireless Sensor Network Deployment for Monitoring Wildlife Passages. Sensors 2010, no. 10, pp. 7236-7262.

Garzón, J., 1977. Birds of prey in Spain. The present situation. World Conference on Birds of Prey. Report of Proceedings, International Council for Bird Preservation, Cambridge, UK. pp. 159-170.

González, J. L. \& Merino, M., 1990. El cernícalo primilla (Falco naumanni) en la Península Ibérica. Situación, Problemática Y Aspectos Biológicos. ICONA, Madrid.

Handcock, R. N., Swain, D. L., Bishop-Hurley, G. J., Patison, K. P., Wark, T., Valencia, P., Corke, P., O’Neill, C. J., 2009. Monitoring Animal Behaviour and Environmental Interactions Using Wireless Sensor Networks, GPS Collars and Satellite Remote Sensing. Sensors no. 9, pp. 3586-3603

Hwang, J., Shin, C., Yoe, H., 2010. Study on an Agricultural Environment Monitoring Server System using Wireless Sensor Networks. Sensors no. 10, pp. 11189-11211.

Jang, R. J-S., 1993. ANFIS: Adaptive-Network-Based Fuzzy Inference System. IEEE Transactions on Systems, Man, and Cybernetics, vol. 23, pp. 665-685.

Kohonen, T., 1990. The self-organizing map. Proceedings of the IEEE, vol 78 (9), pp. 1464-1480.

Kokko, H., Wong, B. B. M., 2007. What determines sex roles in mate searching? Evolution no. 61. pp. 11621175.

Maynard Smith, J., Price, G. R., 1973. The logic of animal conflict. Nature 246. pp.15-8

Newton, I., 1992. Lifetime reproduction in birds. Academic Press, London.

Peet, N. B. and Gallo-Orsi, U., 2000. Action plan for the Lesser Kestrel Falco naumanni. Council of Europe and BirdLife International, Cambridge, U.K.

Polastre, J., Szewczyk, R. and Culler, D., 2005. Telos: Enabling ultra-low power wireless research. 4th Int. Symp. on Information Processing in Sensor Networks (IPSN), Los Angeles, CA, April, pp. 364-369.

Rodríguez, C., and Bustamante, J., 2003. The effect of weather on Lesser Kestrel breeding success: can climate change explain historical population declines? Journal of Animal Ecology no. 72. pp.793-810.

Schlomer, G. L., Ellis, B. J., Garber, J., 2010. MotherChild Conflict and Sibling Relatedness: A Test of Hypotheses From Parent-Offspring Conflict Theory. Journal of Research on Adolescence no. 20. pp. 287 306.

Sridhar, P., 2007. Hierarchical aggregation and intelligent monitoring and control in fault-tolerant wireless sensor networks. University of New Mexico, 2007.

Trivers, R.L., 1974. Parent-offspring conflict. Am. Zool. No. 14. pp 249-264.

Valente, J., Sanz, D., Barrientos, A., Cerro, J., Ribeiro, A., Rossi, C., 2011. An Air-Ground Wireless Sensor Network for Crop Monitoring. Sensors no. 11, pp. 6088-6108. 